SISSA/86/2003/EP

SLAC-PUB-10189

hep-ph/0310055

October 31, 2018

\title{
Effects of the Neutrino $B$-term on Slepton Mixing and Electric Dipole Moments*
}

\author{
Yasaman Farzan \\ Stanford Linear Accelerator Center, 2575 Sand Hill Road, Menlo Park, CA 94025 \\ and \\ Scuola Internazionale superiore di Studi Avanzati, via Beirut 4, I-34014 Trieste, Italy \\ email:yasaman@slac.stanford.edu
}

\begin{abstract}
The supersymmetric standard model with right-handed neutrino supermultiplets generically contains a soft supersymmetry breaking mass term: $\delta L=\frac{1}{2} B_{\nu} M \tilde{\nu}_{R} \tilde{\nu}_{R}$. We call this operator the "neutrino $B$-term". We show that the neutrino $B$-term can give the dominant effects from the neutrino sector to lepton flavor violating processes and to lepton electric dipole moments.
\end{abstract}

\section{Introduction}

The minimal supersymmetric standard model (MSSM), like the standard model itself, predicts zero mass for neutrinos, and this is not compatible with the recent neutrino observations. One of the most promising methods to attribute tiny but non-zero mass to neutrinos is the seesaw mechanism [1], which requires three extremely heavy right-handed neutrinos. In the MSSM with right-handed neutrino supermultiplets, leptonic part of the superpotential is

$$
W=Y_{l}^{i j} \epsilon_{\alpha \beta} H_{1}^{\alpha} l_{R i}^{c} L_{j}^{\beta}+Y_{\nu}^{i j} \epsilon_{\alpha \beta} H_{2}^{\alpha} \nu_{R i} L_{j}^{\beta}+\frac{1}{2} M_{i j} \nu_{R i} \nu_{R j},
$$

where $L_{j}^{\beta}$ is the supermultiplet corresponding to the doublet $\left(\nu_{L j}, l_{L j}\right)$. Without loss of generality, we can rephase and rotate the fields to make the matrices $Y_{l}^{i j}$ and $M_{i j}$ real and

*Work supported by the U. S. Department of Energy under contract DE-AC03-76SF00515. 
diagonal: $Y_{l}^{i j}=\operatorname{diag}\left(Y_{e}, Y_{\mu}, Y_{\tau}\right)$ and $M^{i j}=\operatorname{diag}\left(M_{1}, M_{2}, M_{3}\right)$. In this basis, $Y_{\nu}$ can have off-diagonal and complex elements. Soft supersymmetry breaking terms of the Lagrangian in the context of this model can include

$$
-\mathcal{L}_{\tilde{\nu}_{R}}^{s o f t}=\left(m_{0}^{2}\right)_{j}^{i}\left(\tilde{\nu}_{R}^{i}\right)^{\dagger} \tilde{\nu}_{R}^{j}+\left[\frac{1}{2} B_{\nu} M^{i j} \tilde{\nu}_{R}^{i} \tilde{\nu}_{R}^{j}+\text { h.c. }\right] .
$$

The second term in Eq. (2), the "neutrino B-term", is a lepton-number-violating term which can cause profound effects including sneutrino-antisneutrino oscillation $[2,3]$. The parameter $B_{\nu}$ is allowed to be much larger than the electroweak scale because it is only associated with $\tilde{\nu}_{R}$, which is a electroweak singlet. It has been shown that a nonzero neutrino $B$-term can create neutrino mass through one-loop diagrams [2]. The upper bound on neutrino mass can then be translated into a bound on $B_{\nu}$

$$
B_{\nu}<10^{3} m_{0}
$$

If this bound is saturated, some new effects are expected both in the $e^{+} e^{-}$accelerator experiments [2] and in cosmology [3]. In particular, the large values of $B_{\nu}$ can induce slepton-antislepton oscillation. In this paper, we show that large values of $B_{\nu}$ can also affect other observables.

It is well-known that non-zero flavor-number-violating slepton mass terms in the soft Lagrangian $\left(m_{\alpha \beta}^{2} \tilde{L}_{\alpha}^{\dagger} \tilde{L}_{\beta} \quad \alpha \neq \beta\right)$ can give rise to rare decays such as $(\mu \rightarrow \gamma e),(\tau \rightarrow \gamma e)$ and $(\tau \rightarrow \gamma \mu)$. One way to avoid flavor changing neutral current (FCNC) effects is to choose the off-diagonal mass terms to be small. In fact, theories such as mSUGRA suggest that at the GUT scale, the soft supersymmetry breaking terms are flavor blind; that is at the GUT scale

$$
\begin{aligned}
-\mathcal{L}_{\text {soft }} & =m_{0}^{2}\left(\tilde{L}_{L \alpha}^{\dagger} \tilde{L}_{L \alpha}+\tilde{l}_{R \alpha}^{\dagger} \tilde{l}_{R \alpha}+\tilde{\nu}_{R \alpha}^{\dagger} \tilde{\nu}_{R \alpha}+H_{1}^{\dagger} H_{1}+H_{2}^{\dagger} H_{2}\right)+\frac{1}{2} m_{1 / 2}\left(\tilde{B}^{\dagger} \tilde{B}+\tilde{W}^{a^{\dagger}} \tilde{W}^{a}\right) \\
& +\left(b H_{1} H_{2}+\text { h.c. }\right)+a_{0}\left(Y_{l}^{i j} \epsilon_{\alpha \beta} H_{1}^{\alpha} \tilde{l}_{R i}^{\dagger} \tilde{L}_{L j}^{\beta}+Y_{\nu}^{i j} \epsilon_{\alpha \beta} H_{2}^{\alpha} \tilde{\nu}_{R i} \tilde{L}_{L j}^{\beta}\right) \\
& +\left(\frac{1}{2} B_{\nu} M_{i} \tilde{\nu}_{R}^{i} \tilde{\nu}_{R}^{i}+\text { h.c. }\right)
\end{aligned}
$$

with universal $m_{0}^{2}, m_{1 / 2}$ and $a_{0}$.

Due to the off-diagonal elements of the neutrino Yukawa coupling, running from the GUT scale down to the electroweak scale produces non-vanishing off-diagonal mass terms for the left-handed slepton doublet:

$$
m_{(1) \alpha \beta}^{2}=-\sum_{k} \frac{Y_{\nu}^{k \alpha}\left(Y_{\nu}^{k \beta}\right)^{*}}{16 \pi^{2}}\left[6 m_{0}^{2}\left(\log \frac{\Lambda_{G U T}}{M_{k}}-1\right)+a_{0}^{2}\left(2 \log \frac{\Lambda_{G U T}}{M_{k}}-1\right)\right] .
$$


The leading-log part has first been noticed and studied in [4] and then worked out in a series of papers (e.g. see [5]). However, the effect of the neutrino $B$-term on running of parameters has been ignored. In section 2 , we study this effect and show that if $B_{\nu}$ is large, its contribution will dominate over the effects in Eq. (5).

In the MSSM with flavor blind soft supersymmetry breaking terms, besides the phases in Yukawa couplings, there are two other independent CP-violating phases, usually chosen to be the phases of $a_{0}$ and $\mu$ parameters. These phases can create electric dipole moments (EDMs) for charged leptons and for the neutron [6]. In the presence of the neutrino $B$-term, there is one more phase which can also give a contribution to the EDM of charged leptons. In section 3 , we show that even if at the GUT scale, no $A$-term is present $\left(a_{0}=0\right)$, through 1-loop corrections, the neutrino $B$-term creates $A$-terms for leptons at the electroweak scale. This effect could be the dominant term in lepton EDMs.

In section 4, we explore the upper bounds on imaginary and real parts of $B_{\nu}$ resulting from the upper bounds on the branching ratios of the rare decays $\left(\operatorname{BR}\left(l_{\alpha} \rightarrow l_{\gamma}+\gamma\right)\right)$ and the EDMs of the charged leptons. The main limitation will be the uncertainty in the pattern of neutrino Yukawa couplings, $Y_{\nu}$.

\section{Effects of the neutrino $B$-term on slepton mixing}

It has been shown that the off-diagonal slepton masses $\left(m_{\alpha \beta}^{2} \tilde{L}_{L \alpha}^{\dagger} \tilde{L}_{L \beta} \alpha \neq \beta\right)$ at one-loop level can give rise to lepton-number-violating rare decays such as $(\mu \rightarrow e \gamma),(\tau \rightarrow \mu \gamma)$ and $(\tau \rightarrow e \gamma)[5,7]$. In the mass insertion approximation, a simplified formula can be derived $[8]:$

$$
\operatorname{Br}\left(l_{\alpha} \rightarrow l_{\beta}+\gamma\right) \sim \frac{\alpha^{3}}{G_{F}^{2}} \frac{\left|m_{\alpha \beta}^{2}\right|^{2}}{m_{\text {susy }}^{8}} \tan ^{2} \beta .
$$

The upper bounds on the branching ratios of the rare decays [9] can be interpreted as bounds on the off-diagonal elements of $\left|m_{\alpha \beta}^{2}\right|$ :

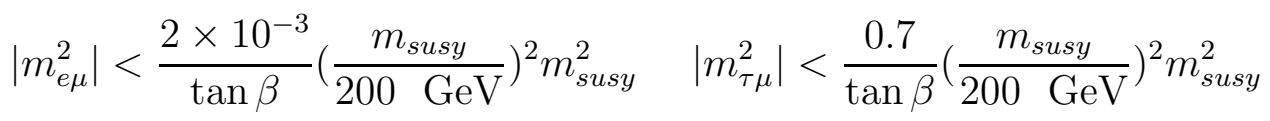

and

$$
\left|m_{\tau e}^{2}\right|<\frac{1}{\tan \beta}\left(\frac{m_{\text {susy }}}{200 \mathrm{GeV}}\right)^{2} m_{\text {susy }}^{2} .
$$

The next generation of experiments [10] is expected to improve (7) to

$$
\left|m_{\text {e }}^{2}\right|<\frac{6 \times 10^{-5}}{\tan \beta}\left(\frac{m_{\text {susy }}}{200 \mathrm{GeV}}\right)^{2} m_{\text {susy }}^{2} \quad\left|m_{\tau \mu}^{2}\right|<\frac{0.02}{\tan \beta}\left(\frac{m_{\text {susy }}}{200 \mathrm{GeV}}\right)^{2} m_{\text {susy }}^{2} .
$$


The off-diagonal mass terms for left-handed sleptons receive contribution from the neutrino $B$-term through the two diagrams shown in Fig. 1. The two lepton number violating vertices on the neutrino line are the neutrino $B$-term and the standard $\tilde{\nu}_{R}$ mass term. The neutrino $A$-term is also needed. The amplitude corresponding to diagram (a) is equal to

$$
\begin{aligned}
-i \mathcal{M} & =\sum_{k} i Y_{\nu}^{k \alpha} i\left(a_{0} Y_{\nu}^{k \beta}\right)^{*} \int \frac{d^{4} k}{(2 \pi)^{4}} \frac{i}{k^{2}} \frac{i}{k^{2}-M_{k}^{2}}\left(-i B_{\nu} M_{k}\right) \frac{i}{k^{2}-M_{k}^{2}} i M_{k} i \\
& =\sum_{k} \frac{i}{(4 \pi)^{2}} a_{0}^{*} Y_{\nu}^{k \alpha}\left(Y_{\nu}^{k \beta}\right)^{*} B_{\nu} .
\end{aligned}
$$

Similarly, diagram (b) gives

$$
-i \mathcal{M}=\sum_{k} \frac{i}{(4 \pi)^{2}} a_{0} Y_{\nu}^{k \alpha}\left(Y_{\nu}^{k \beta}\right)^{*} B_{\nu}^{*}
$$

The mass correction is given by the sum of the two amplitudes:

$$
m_{(2) \alpha \beta}^{2}=-2 \sum_{k} \frac{Y_{\nu}^{k \alpha}\left(Y_{\nu}^{k \beta}\right)^{*} \operatorname{Re}\left[a_{0} B_{\nu}^{*}\right]}{(4 \pi)^{2}},
$$

which has to be added to $m_{(1) \alpha \beta}^{2}$ presented in Eq. (5). Note that the contribution we have found does not depend on the heavy right-handed masses at all. This can be traced back to the form of the neutrino $B$-term assumed in Eq. (4). Had we defined this term as $B_{\nu}^{2} \tilde{\nu}_{R} \tilde{\nu}_{R}$, the result would have been proportional to $\operatorname{Re}\left[a_{0}\left(B_{\nu}^{2}\right)^{*}\right] / M_{k}$.

Up to the factors of $\log \left(\Lambda_{G U T} / M_{k}\right), m_{(1) \alpha \beta}^{2}$ and $m_{(2) \alpha \beta}^{2}$ (see Eqs. $\left.(5,12)\right)$ have the same flavor structure. The structure can be different only if the masses of right-handed neutrinos are hierarchical $\left(M_{1} \ll M_{2} \ll M_{3}\right)$. Although the one-loop mass matrix presented in Eq. (5) is enhanced by a factor of $\left(6 \log \left(\Lambda_{G U T} / M_{k}\right) \sim 10\right)$, the neutrino $B$-term contributions given in Eq. (12) dominates if $B_{\nu} \sim 10^{3} m_{0}$ as allowed by (3).

The dependence of $m_{(2) \alpha \beta}^{2}$ on $B_{\nu}$ is through the combinations $\sum_{k} Y_{\nu}^{k \alpha}\left(Y_{\nu}^{k \beta}\right)^{*}$. If these combinations are so large that masses in Eq. (5) saturate the bounds $(7,8), B_{\nu}$ has to be smaller than $10 m_{0}$ (to be compared with Eq. (3)). However, to derive conclusive bounds on $B_{\nu}$, first, we have to find some lower bounds on the $\sum_{k} Y_{\nu}^{k \alpha}\left(Y_{\nu}^{k \beta}\right)^{*}$ combinations. Unfortunately, such piece of information is not available at the moment. In section 4 , We will discuss this further.

In the discussion above, we have assumed $a_{0} \sim m_{0} \sim m_{\text {susy }}$ but it is possible that $a_{0}$ is much smaller than $m_{0}$. In this case, $m_{(2) \alpha \beta}^{2}$ given in Eq. (12) will not be the dominant effect. At the one loop level, there is no contribution to $m_{\alpha \beta}^{2}$ proportional to $\left|B_{\nu}\right|^{2}$ : it can be shown that the two one-loop diagrams which are proportional to $\left|B_{\nu}\right|^{2}$ (depicted in Fig. 2) cancel each other at zero external momentum. However, at the two-loop level, there is a 
contribution proportional to $\left|B_{\nu}\right|^{2}$ which can dominate over $m_{(1) \alpha \beta}^{2}$ (Eq. (5)) provided that $\left|B_{\nu}\right|^{2} Y_{\nu} Y_{\nu}^{*} /(4 \pi)^{2}>m_{0}^{2}$

\section{Effects of the neutrino $B$-term on $A$-terms}

In this section, we show that the neutrino $B$-term creates $A$-term for leptons through 1-loop diagrams. We then discuss how this will affect EDMs.

As is discussed in the literature [6], the phases of $\mu$ and $a_{0}$ can create electric dipole moments for charged leptons as well as for the neutron. The current bounds on lepton EDMs are

$$
d_{e}<1.5 \times 10^{-27} \text { e cm [16] } d_{\mu}<7 \times 10^{-19} \text { e } \mathrm{cm} \quad[9]
$$

and

$$
d_{\tau}<3 \times 10^{-16} \text { e cm }[9]
$$

Proposed future experiments are expected to set stronger bounds:

$$
d_{e}<10^{-32} \text { e cm [17] } d_{\mu}<10^{-24}\left(5 \times 10^{-26}\right) \text { e cm [18] ([19]) }
$$

The bounds on the electric dipole moments of the charged leptons yield strong bounds on the imaginary parts of $\mu$ and $a_{0}$ [20].

The phase of the neutrino $B$-term can provide yet another source of CP-violation. ${ }^{\dagger}$ When we fixed mass matrix $M$ to be real, the phases of $\tilde{\nu}_{R}$ were fixed; therefore, the phase of $B_{\nu}$ in this convention cannot be removed. We expect the imaginary part of $B_{\nu}$ to give contribution to EDMs.

The parameter $B_{\nu}$ contributes to the $A_{l}$-term through the diagram shown in Fig. 3. Adding this correction to the tree level $A_{l}$ at the GUT scale (see Eq. (4)), we find that at the electroweak scale,

$$
\begin{aligned}
-i A_{l}^{j i} & =-i a_{0} Y_{l}^{j i} \delta_{i j}+\left(i Y_{l}^{j j}\right)(i) i\left(Y_{\nu}^{k j}\right)^{*}\left(i Y_{\nu}^{k i}\right) \int \frac{i}{k^{2}} \frac{i}{k^{2}-M_{k}^{2}}\left(-i B_{\nu} M_{k}\right) \frac{-i M_{k}}{k^{2}-M_{k}^{2}} \frac{d^{4} k}{(2 \pi)^{4}} \\
& =-i a_{0} Y_{l}^{j i} \delta_{i j}-\frac{i}{(4 \pi)^{2}} Y_{l}^{j j}\left(Y_{\nu}^{k j}\right)^{*} Y_{\nu}^{k i} B_{\nu} .
\end{aligned}
$$

Similarly, the neutrino $B$-term contributes to the the $A_{\nu}$-term through the diagram shown in Fig. 4 gives:

$$
-i A_{\nu}^{k i}=-i a_{0} Y_{\nu}^{k i}+\left(i Y_{\nu}^{q i}\right)(i) i\left(Y_{\nu}^{q j}\right)^{*}\left(i Y_{\nu}^{k j}\right) \int \frac{i}{k^{2}} \frac{i}{k^{2}-M_{q}^{2}}\left(-i B_{\nu} M_{q}\right) \frac{-i M_{q}}{k^{2}-M_{q}^{2}} \frac{d^{4} k}{(2 \pi)^{4}}
$$

${ }^{\dagger}$ Within the extended MSSM, the Yukawa couplings $\left(Y_{\nu}\right)$ are another source of CP-violation. However, it can be shown that up to two-loop level, Yukawa couplings do not yield any contribution to the EDMs [21]. 


$$
=-i a_{0} Y_{\nu}^{k i}-\frac{i}{(4 \pi)^{2}} Y_{\nu}^{q i}\left(Y_{\nu}^{q j}\right)^{*} Y_{\nu}^{k j} B_{\nu}
$$

According to [20], for $m_{0} \sim 200 \mathrm{GeV}$, the present bound $\left(d_{e}<10^{-27} \mathrm{e} \mathrm{cm}\right)$ implies

$$
\operatorname{Im}\left(A_{l}^{e e}\right)\left\langle H_{1}\right\rangle /\left(m_{e} m_{0}\right) \lesssim 0.1
$$

Since the dependence of $d_{e}$ on $\operatorname{Im}\left(A_{l}^{e e}\right)$ is linear, if in the future the bound $d_{e}<10^{-32}$ e cm is obtained, the above bound will be improved to

$$
\operatorname{Im}\left(A_{l}^{e e}\right)\left\langle H_{1}\right\rangle /\left(m_{e} m_{0}\right) \lesssim 10^{-6}
$$

Assuming that $Y_{\nu}^{k e}\left(Y_{\nu}^{k e}\right)^{*} \sim 1$, the present bound (18) gives $\operatorname{Im}\left[B_{\nu}\right]<10 m_{0}$ (we have used Eq. (16) and $\left.m_{e}=\left\langle H_{1}\right\rangle Y_{l}^{e e}\right)$. Future experiments can make the bound dramatically stronger.

Discovery of a lepton EDM could provide invaluable information on $B_{\nu}$. The neutrino $B$-term gives a contribution to $A_{l}$; however, it has no impact on the $A$-term of quarks. As a result, $\operatorname{Im}\left(B_{\nu}\right)$ will not affect the EDM of the neutron. On the other hand, $\operatorname{Im}\left(a_{0}\right)$ and $\operatorname{Im}(\mu)$ give contribution both to the EDM of charged lepton and neutron. If $d_{e}$ turns out to be non-zero while $d_{n} \ll d_{e}$, we will have a strong evidence in favor of a non-zero complex $B_{\nu}$. Synergy between the rare decay experiments and EDM searches can help to determine the parameters involved.

There is another point which is noteworthy: EDMs are proportional to $\left|Y_{\nu}^{k \alpha}\right|^{2}$, and $\operatorname{Br}\left(l_{\alpha} \rightarrow l_{\beta} \gamma\right)$ are given by $\left|Y_{\nu}^{k \alpha}\left(Y_{\nu}^{k \beta}\right)^{*}\right|$, which both are independent of $M_{l}$. To the author's best knowledge, there is no other observable that depends on these combinations. If $\left|B_{\nu}\right|$ is large, by studying these observables we can extract additional information on Yukawa couplings which will improve our current understanding of the seesaw mechanism and leptogenesis.

If the neutrino $B$-term gives the dominant contribution to the electric dipole moments, we expect $d_{\tau} /\left(m_{\tau} \sum_{k}\left|Y_{\nu}^{k \tau}\right|^{2}\right)=d_{\mu} /\left(m_{\mu} \sum_{k}\left|Y_{\nu}^{k \mu}\right|^{2}\right)=d_{e} /\left(m_{e} \sum_{k}\left|Y_{\nu}^{k e}\right|^{2}\right)$; therefore, if $d_{e}$ is close to its present upper bound, $d_{e} \sim 10^{-27} \mathrm{e} \mathrm{cm}$, we expect $d_{\mu} \sim 10^{-25} \mathrm{e} \mathrm{cm} \mathrm{which} \mathrm{can} \mathrm{be}$ tested in experiments proposed [19].

\section{Bounds on $B_{\nu}$}

In sections 2 and 3, we have shown that large values of $B_{\nu}$ can lead to flavor-violating rare decays and EDMs of charged leptons. However, the dependence of these observables on $B_{\nu}$ is through the unknown combination of Yukawa couplings $Y_{\nu}^{k \alpha}\left(Y_{\nu}^{k \beta}\right)^{*}$. To derive upper bounds on $B_{\nu}$, we have to find other observables that provide lower bounds on these 
combinations. In this section, we combine the information on the Yukawa couplings from different observations to derive a lower bound on the factors $Y_{\nu}^{k \alpha}\left(Y_{\nu}^{k \beta}\right)^{*}$. We will then use the current upper bounds on the branching ratios of the rare decays and the values of EDMs to extract upper bounds on $B_{\nu}$.

Neutrino masses depend on the Yukawa couplings through

$$
m_{\alpha \beta}^{(\nu)}=\sum_{k} Y_{\nu}^{k \alpha} \frac{1}{M_{k}} Y_{\nu}^{k \beta}\left\langle H_{2}\right\rangle^{2} .
$$

Currently we only have bounds on the neutrino masses [11]:

$$
\sqrt{\Delta m_{a t m}^{2}}<\sum m_{\nu}<1 \mathrm{eV}
$$

where $\Delta m_{\text {atm }}^{2}=2.5 \times 10^{-3} \mathrm{eV}^{2}[12]$. Future terrestrial and cosmological experiments will improve these bounds. Our knowledge of the masses of the right-handed neutrinos $\left(M_{k}\right)$ is even less complete than the information on $m_{\alpha \beta}^{(\nu)}$. If leptogenesis is the mechanism behind the Baryon asymmetry of the universe [13],

$$
M_{k}>6 \times 10^{8} \mathrm{GeV} \text {. }
$$

Assuming $m_{\alpha \beta}^{(\nu)} \sim 0.1 \mathrm{eV}$, at least for one $k$,

$$
Y_{\nu}^{k \alpha}\left(Y_{\nu}^{k \beta}\right)^{*}>\left(\frac{m_{\alpha \beta}^{(\nu)}}{0.1 \mathrm{eV}}\right) \frac{2 \times 10^{-6}}{\sin ^{2} \beta}
$$

where we have used the bound in Eq. (21).

The parameter $m_{e e}^{(\nu)}$ can be extracted directly from neutrinoless double decay observations. If $m_{e e}^{(\nu)} \sim 0.1 \mathrm{eV}$, its effect should be observable in future experiments [14]. Using Eq. (16), $d_{e}<10^{-27}$ e cm (see Eq. (18)) yields

$$
\frac{\operatorname{Im}\left(B_{\nu}\right)}{m_{0}}<10^{7}\left(\frac{0.1 \mathrm{eV}}{m_{e e}^{(\nu)}}\right)
$$

where we have used Eqs. (16,18). In future, if the bound $d_{e}<10^{-32}$ is obtained, this bound will be improved by five orders of magnitude (see Eq. (19)) which means it can be more restrictive than the bound in Eq. (3).

Extracting bounds on $Y_{\nu}^{k \alpha}\left(Y_{\nu}^{k \beta}\right)^{*} \alpha \neq \beta$ needs un-testable assumptions because, unlike $m_{e e}^{(\nu)}, m_{\alpha \beta}^{(\nu)} \alpha \neq \beta$ cannot be directly measured. Even if forthcoming experiments find that the overall neutrino mass is of order of $0.1 \mathrm{eV}$ (quasi-degenerate mass scheme), it will be difficult to derive definite lower bounds on $\left[m_{\alpha \beta}^{(\nu)} \quad(\alpha \neq \beta)\right]$. In the case of quasi-degenerate 
mass scheme (the scheme for which the absolute values of mass eigenvalues are much larger than $\left.\sqrt{\Delta m_{a t m}^{2}}\right)$ with zero Majorana phases we expect $m_{e \mu}^{(\nu)}, m_{\mu \tau}^{(\nu)}, m_{e \tau}^{(\nu)} \ll m_{e e}^{(\nu)}, m_{\mu \mu}^{(\nu)}, m_{\tau \tau}^{(\nu)}$. Only in the framework of quasi-degenerate neutrino mass scheme with at least one non-zero Majorana phase, large off-diagonal neutrino masses, $m_{e \mu}^{(\nu)}, m_{\mu \tau}^{(\nu)}, m_{e \tau}^{(\nu)} \gg \sqrt{\Delta m_{a t m}^{2}}$ are possible (a phase equal to $\pi$ also works). On the other hand, determining the values of Majorana phases is very challenging, if possible at all [15]. Nevertheless, let us suppose $m_{e \mu}^{(\nu)} \sim 0.1 \mathrm{eV}$. Then, assuming that the factors $Y_{\nu}^{k e}\left(Y_{\nu}^{k \mu}\right)^{*}$ do not cancel each other, the present bound on $\left|m_{e \mu}^{2}\right|$ given in Eq. (7) implies

$$
\operatorname{Re}\left[a_{0} B_{\nu}^{*}\right] / m_{0}^{2}<\frac{10^{5}}{\tan \beta}\left(\frac{m_{0}}{200 \mathrm{GeV}}\right)^{2}\left(\frac{0.1 \mathrm{eV}}{m_{e \mu}^{(\nu)}}\right) .
$$

The future possible bounds (inferred from Eq. (9)) can be more restrictive than Eq. (3):

$$
\operatorname{Re}\left[a_{0} B_{\nu}^{*}\right] / m_{0}^{2}<\frac{3 \times 10^{3}}{\tan \beta}\left(\frac{m_{0}}{200 \mathrm{GeV}}\right)^{2}\left(\frac{0.1 \mathrm{eV}}{m_{e \mu}^{(\nu)}}\right) ;
$$

however, it will suffer from the untestable assumptions we have already discussed.

\section{Concluding remarks}

We have studied the effects of the neutrino $B$-term on the slepton mixing and EDMs of charged leptons in the framework of seesaw model embedded in the MSSM with universal soft supersymmetry breaking terms.

If $B_{\nu}>10 m_{0} \sim 10 a_{0}$ but $a_{0}>B_{\nu} Y_{\nu}\left(Y_{\nu}\right)^{*} /(4 \pi)^{2}$, the dominant flavor-violating slepton masses are given by Eq. (12) rather than Eq. (5). For values of $B_{\nu}$ satisfying $B_{\nu} Y_{\nu}\left(Y_{\nu}\right)^{*} /(4 \pi)^{2}>$ $a_{0}$ and $\left|B_{\nu}\right|^{2} Y_{\nu}\left(Y_{\nu}\right)^{*} /(4 \pi)^{2}>m_{0}^{2}$, the two-loop contribution proportional to $\left|B_{\nu}\right|^{2}$ can be dominant. The bounds on Yukawa couplings and neutrino $B$-term which have been discussed in the literature allow quite large contributions to the slepton masses, violating the upper bounds from rare flavor-violating decays. However, since there is no direct lower bound on the combinations of neutrino Yukawa couplings appearing in the formulations, it is not possible to derive any upper bound on the $\operatorname{Re}\left[a_{0} B_{\nu}^{*}\right]$ without untestable assumptions.

The parameter $B_{\nu}$ can be considered as another source for CP-violation and therefore EDMs. In fact, we have shown that neutrino $B$-term directly creates $A$-terms both for neutrinos and charged leptons- but not quarks- even if $a_{0}=0$ at the GUT scale. The imaginary part of $A_{l}$ gives a contribution to the EDMs of charged leptons. If the $B_{\nu}$ effect is dominant, we expect $d_{\tau} /\left(m_{\tau} \sum_{k}\left|Y_{\nu}^{k \tau}\right|^{2}\right)=d_{\mu} /\left(m_{\mu} \sum_{k}\left|Y_{\nu}^{k \mu}\right|^{2}\right)=d_{e} /\left(m_{e} \sum_{k}\left|Y_{\nu}^{k e}\right|^{2}\right)$; therefore, if $d_{e}$ is close to its present upper bound we expect that the proposed experiments [19] will 
be able to measure the value of $d_{\mu}$. Discovery of non-zero $d_{e}$ and $d_{\mu}$ while $d_{n} \ll d_{e}$ would be strongly suggestive of large $\operatorname{Im}\left(B_{\nu}\right)$. In this case, $d_{e} \propto \sum_{k} Y_{\nu}^{k e}\left(Y_{\nu}^{k e}\right)^{*} \operatorname{Im}\left(B_{\nu}\right) \cdot \operatorname{If} \operatorname{Im}\left(B_{\nu}\right)$ is determined by some other observations, information on $d_{e}$ and $\operatorname{Im}\left(B_{\nu}\right)$ combined with $m_{e e}^{(\nu)}=\sum_{k}\left(Y_{\nu}^{k e}\right)^{2} / M_{k}$ (extracted from neutrinoless double beta decay searches) can provide us with information on the values of $M_{k}$, shedding light on the origin of neutrino masses and on leptogenesis.

\section{Acknowledgment}

I would like to thank Y. Grossman, M. Peskin and A. Yu. Smirnov for useful discussions and encouragement. I am also grateful to M. M. Sheikh-Jabbari for careful reading of the manuscript.

\section{References}

[1] T. Yanagida, Proceeding of Workshop on Unified Theory and Baryon Number of the Universe, eds.; O. Sawada and A. Sugamoto (KEK, 1979) p. 95; M. Gell-Mann, P. Ramond and R. Slansky, in Supergravity, eds. P. van Niewwnhuizen and D. Freedman (North Holland, Amsterdam, 1979).

[2] Y. Grossman and H. E. Haber Phys. Rev. Lett. 78 (1997) 3438.

[3] L. J. Hall, T. Moroi and H. Murayama, Phys. Lett. B424 (1998) 305; Y. Grossman, T. Kashti, Y. Nir and E. Roulet, hep-ph/0307081; G. D’Ambrosio, G. F. Giudice and M. Raidal, hep-ph/0308031.

[4] F. Borzumati and A. Masiero, Phys. Rev. Lett. 57 (1986) 961.

[5] J. Hisano, T. Moroi, K. Tobe and M. Yamaguchi, Phys. Rev. D53 (1996) 2442.

[6] T. Moroi, Phys. Rev. D 53 (1996) 6565; Erratum-ibid. D 56 (1997) 4424; S. Pokorski, J. Rosiek and C. A. Savoy, Nucl. Phys. B 570 (2000) 81; J. L. Feng, K. T. Matchev and Y. Shadmi, Nucl. Phys. B 613 (2001) 366; M. Garena, et. al., Phys. Lett.B 390 (1997) 234; E. Gabrielli and U. Sadrid, Phys. Rev. Lett.79 (1997) 4752; J. L. Feng and K. T. Matchev, Phys. Rev. Lett.86 (2001) 3480; L. Everett et. al., Phys. Rev. Lett.86 (2001) 3484; T. Ibrahim et. al., Phys. Rev.D 64 (2001) 016010; J. Ellis et. al., Phys. Lett.B 507 (2001) 224; Z. Chacko and G. D. Kribs Phys. Rev.D 64 (2001) 75015; D. G. 
Gerdeno et. al., Phys. Rev.D 64 (2001) 093012; U. Chattopadhyay and P. Nath, Phys. Rev. D 66 (2002) 093001; S. P. Martin and J. D. Wells, Phys. Rev. D 67 (2003) 015002; T. Ibrahim and P. Nath, Phys. Rev.D 58 (1998) 111301; T. Falk and K. Olive, Phys. Lett.B 439 (1998) 71; M. Brhlik, G. Good and G. L. Kane, Phys. Rev.D 59 (1999) 115004; U. Chattopadhyay, T. Ibrahim and P. Roy, Phys. Rev. D 64 (2001) 013004; V. Barger et. al., Phys. Rev. D 64 (2001) 056007; S. Abel, S. Khalil and O. Lebedev, Nucl. Phys. B 606 (2001) 151; T. Ibrahim and P. Nath, Phys. Rev. 64 (2001) 093002 .

[7] J. Hisano and D. Nomura, Phys. Rev. D59 (1999) 116005.

[8] J. A. Casas and A. Ibarra, hep-ph/0109161;

[9] K. Hagiwara at. al., Phys. Rev. D 66 (2002) 010001.

[10] L. M. Barkov at. al., proposal for an experiment at PSI, http://meg.web.psi.ch. I. Hinchliffe and F. E. Paige, Phys Rev. D 63 (2001) 115006; D. F. Carvalho et. al., hep-ph/0206148; J. Kalinowski, Acta Phys. Polon. B33 (2002) 2613.

[11] S. Hannestad, JCAP 0305:004,2003 [astro-ph/0303076]; J. Bonn et. al., Nucl. Phys. Proc. Suppl. 91 (2001) 273; V. M. Lobashev et. al., Proc. of the Int. Con. Neutrino 2000, Sudbury, Canada; Nucl. Proc. Suppl. 77 (1999) 327; Nucl Phys. Proc. Suppl. 91 (2001) 280; V. M. Lobashev, Prog. Part. Phys. 48 (2002)123.

[12] Super-Kamiokande Collaboration, T. Nakaya, eConf C020620 (2002) SAAT01, [hepex/0209036].

[13] E. Kh. Akhmedov, M. Frigerio and A. Yu. Smirnov, JHEP 0309:021,2003; W. Buchmuller, P. Di Bari and M. Plumacher, Nucl. Phys. B643 (2002) 367; S. Davidson and A. Ibarra, Phys. Lett. B535 (2002) 25.

[14] S. Pirro et. al., Nucl Instrum. Meth. A444 (2000) 71; E. Fiorini et. al., Phys. Rep. 307 (1998) 309; A. Bettini, Nucl. Phys. Proc. Suppl. 100 (2001) 332; H. Ejiri, J. Engel, H. Hazama, P. Krastev, N. Kumodi and R. G. H. Robertson, Phys. Rev. Lett. 85 (2000) 2917; GENIUS Collaboration (H. V. Klapdor-Kleingrothaus et al.,), hep-ph/9910205.

[15] V. Barger, S.L. Glashow, P. Langacker and D. Marfatia, Phys. Lett. B540 (2002) 247; H. Nunokawa, W.J.C. Teves and R. Z. Funchal Phys. Rev. D66 (2002) 093010; F. Feruglio, A. Strumia and F. Vissani, Nucl. Phys. B637 (2002) 345. 
[16] E. D. Commins et. al., Phys. Rev.A 50 (1994) 2960.

[17] S. K. Lamoreaux, nucl-ex/0109014.

[18] R. Garey et. al., Letter of intent to BNL (2000); Y. K. Semertzidis et. al., hepph/0012087.

[19] J. Aysto et. al., hep-ph/0109217.

[20] I. Masina and C.A. Savoy, hep-ph/0211283

[21] Y. Farzan and M. E. Peskin, work in preparation. 


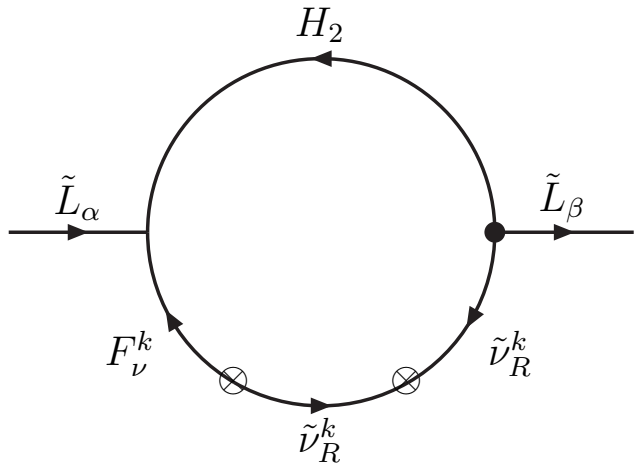

(a)

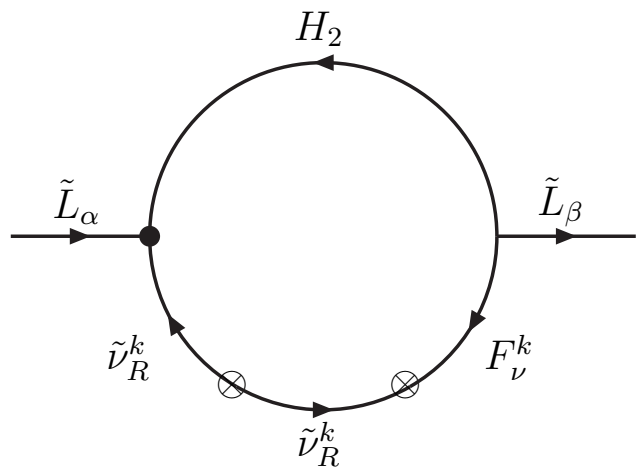

(b)

Figure 1: Diagrams contributing to slepton masses. $F_{\nu}^{k}$ represents the auxiliary field associated with $\tilde{\nu}_{R}^{k}$. The $A_{\nu}$ vertices are marked with black circles.

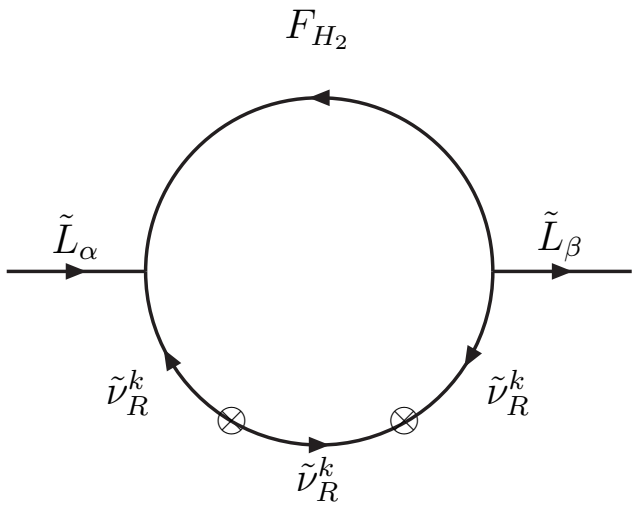

(a)

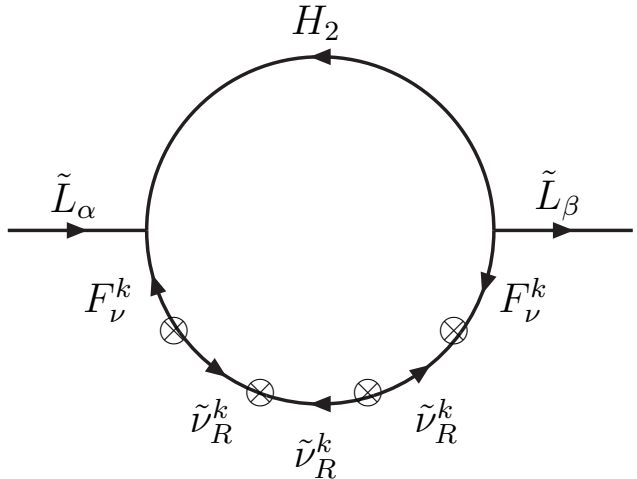

(b)

Figure 2: Diagrams proportional to $|B|^{2}$ contributing to slepton masses. $F_{H_{2}}$ represents the auxiliary field associated with $\mathrm{H}_{2}$. 


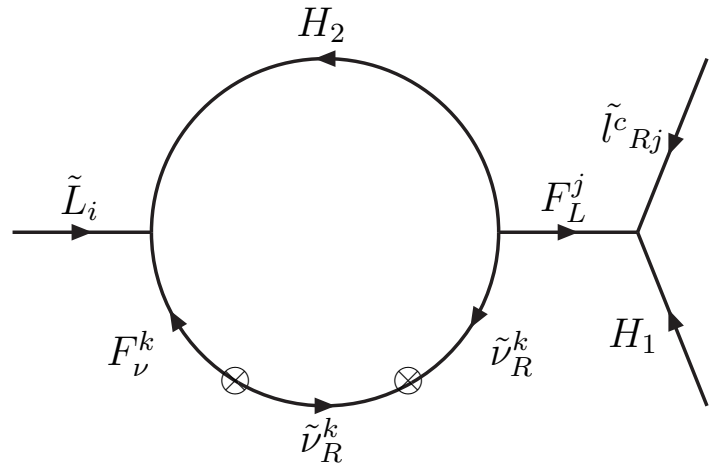

(a)

Figure 3: Diagram contributing to $A_{l} . F_{\nu}^{k}$ and $F_{L}^{j}$ represent the auxiliary fields associated with $\tilde{\nu}_{R}^{k}$ and $\tilde{L}_{j}$, respectively.

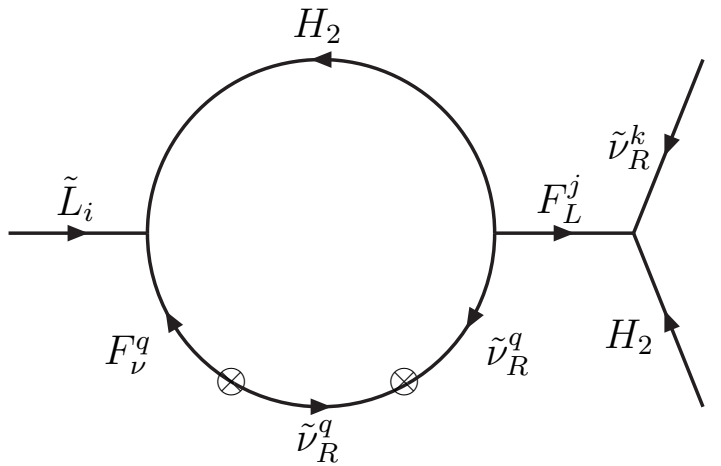

(a)

Figure 4: Diagram contributing to $A_{\nu} . F_{\nu}^{q}$ and $F_{L}^{j}$ represent the auxiliary fields associated with $\widetilde{\nu}_{R}^{q}$ and $\tilde{L}_{j}$, respectively. 